\title{
A Virtual Glovebox for the Digital Enterprise
}

\author{
Christian Seiler, Ralf Dörner \\ Fraunhofer Applications Center for Computer Graphics, D 60486 Frankfurt am Main, Germany \\ \{cseiler,doerner\}@agc.fhg.de
}

\begin{abstract}
The employment of simulation technology together with the exploration of virtual environments promise to be a unique advantage for digital enterprises in diverse fields like planning, rapid prototyping, marketing and presentations, as well as information dissemination and training. However, there are serious obstacles that inhibit digital enterprises to take a competitive advantage when using 3D for business processes. Most of these obstacles are concerned with the fact that 2D interaction metaphors are used for interacting with 3D content. With the motivation to help a digital enterprise to benefit from the advantages of applying $3 \mathrm{D}$ and virtual reality for different purposes and different user groups, this paper introduces an innovative dedicated interaction device for the digital enterprise. It employs the metaphor of a glove box where stereoscopic visualization together with haptic feedback is given in a natural manner. This makes it intuitive to manipulate three-dimensional content and makes it easy for users to navigate and to orient themselves in a virtual 3D world. Beside presenting the Virtual Glove Box device, we will discuss its advantages for new industrial organisations and deal with the question how to integrate it in existing structures and processes of an enterprise.
\end{abstract}

Key words: Virtual Glove Box, Input and Output Devices, Digital Enterprise, ThreeDimensional Content, Virtual Reality, Haptic Feedback

\section{INTRODUCTION}

Economic trends like globalisation lead to new organisation structures of enterprises that depend heavily on digitised information and digital data. The employment of simulation technology together with the exploration of virtual environments [4] promise to be a unique advantage for these digital enterprises in diverse fields like planning, rapid prototyping, marketing and presentations, or information dissemination and training. This is specifically

The original version of this chapter was revised: The copyright line was incorrect. This has been corrected. The Erratum to this chapter is available at DOI: 10.1007/978-0-387-35492-7_50 
true if we focus on $3 \mathrm{D}$ which is important since many products are inherently three-dimensional and their spatial layout is important (e.g. assembly of parts, planning in the field of pipeline construction). However, its up to modern digital enterprises to overcome obstacles in the usage of $3 \mathrm{D}$ in order to take a competitive advantage when using 3D for business processes. Most of these obstacles are concerned with the fact that 2D interaction metaphors are used for interacting with 3D content. This makes it non-intuitive to manipulate three-dimensional content and is a reason why most people find it difficult to navigate and to orient themselves in a virtual $3 \mathrm{D}$ world [3]. In addition, there is no haptic feedback and the 3D world is displayed on a 2D screen although haptic feedback devices as well as stereo systems are more and more available in low priced versions.

With the motivation to help a digital enterprise to benefit from the advantages of applying 3D and virtual reality for different purposes and different user groups, this paper introduces an innovative dedicated interaction device for the digital enterprise. This interaction device overcomes limitations of traditional interaction devices even the ones that are dedicated to 3D. In the paper we will present and describe this interaction device, called Virtual Glove Box. Moreover, we will discuss its advantages for new industrial organisations and deal with the question how to integrate it in existing structures and processes of an enterprise.

The paper is organized as follows. In the next section we present how the Virtual Glove Box device is built and used. In section 3 we will discuss how the Virtual Glove Box can be seamlessly introduced and integrated in a digital enterprise. Finally, section 4 gives an example application of the Virtual Glove Box.

\section{THE VIRTUAL GLOVE BOX INTERACTION DEVICE}

Although there was a constant advance in 3D computer graphics over the recent years, 3D computer graphics had not much impact on the digital enterprise. Most 3D Computer graphics applications stem from CAD products and are often not interactive. Some of the main reason for the lack of 3D computer graphics were its demand for dedicated hardware, the heterogeneity of the hardware used in the enterprise and the lack of adequate $\mathrm{I} / \mathrm{O}$ devices to interact with $3 \mathrm{D}$ graphics.

With the dramatic increase of 3D performance of low cost graphics hardware the first obstacle for 3D graphics in the digital enterprise is about to disappear. The problem of heterogeneity an interaction, however, still 
persists. While we address the heterogeneity problem in chapter 3 , this chapter will focus on the interaction problem.

3D virtual worlds, as they are created by computer graphics applications, display non existing scenes and objects in a way that mimics our real threedimensional world. While the look of such a virtual world can be very convincing, the "feel" of such a world usually is not. In order to interact with a virtual scene the user has to use $\mathrm{I} / \mathrm{O}$ devices as interfaces between the user and the virtual domain. The $\mathrm{I} / \mathrm{O}$ devices most often used for such a task are mouse and keyboard. These devices are best suited for the 2D desktop metaphor which is widely used for 2D applications, but not appropriate for 3D applications. There are several $\mathrm{I} / \mathrm{O}$ devices which improve the navigation in a 3D world, like joysticks or the space mouse, but these devices are limited in their usage when it comes to moving, touching or manipulating virtual objects.

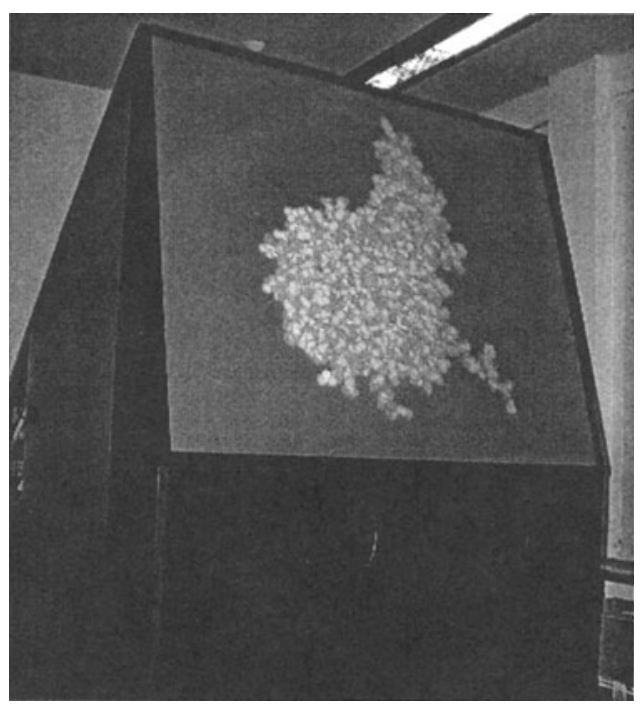

Figure 1: The Virtual Glove Box Device

Another drawback of those devices is that they lack the possibility to display a certain property that most of our real life objects possess intrinsically: Haptic behaviour and force-feedback. While it is commonplace in VR applications to walk or grasp right through solid objects, this is not so in real life. So called haptic or force-feedback devices try to overcome this limitation. But these devices still have several drawbacks, e.g. the PHANToM device [8][9] allows only an indirect manipulation of 3D content, while exo-skeleton devices like the CyberGrasp [1] are not sufficiently integrated in the visual representation of $3 \mathrm{D}$ content. 
In order to overcome the problems and obstacles described above, we introduce a new I/O device called the Virtual Glove Box. A glove box is an apparatus used in chemistry or biology to work on targets in a closed atmosphere, without contaminating the substances or endangering the user. A glove box usually consists of a transparent hull in which the experiments can be performed. The user can reach into the box through gloves which are attached at the inside of the box. The gloves can be reached through corresponding holes in the box.
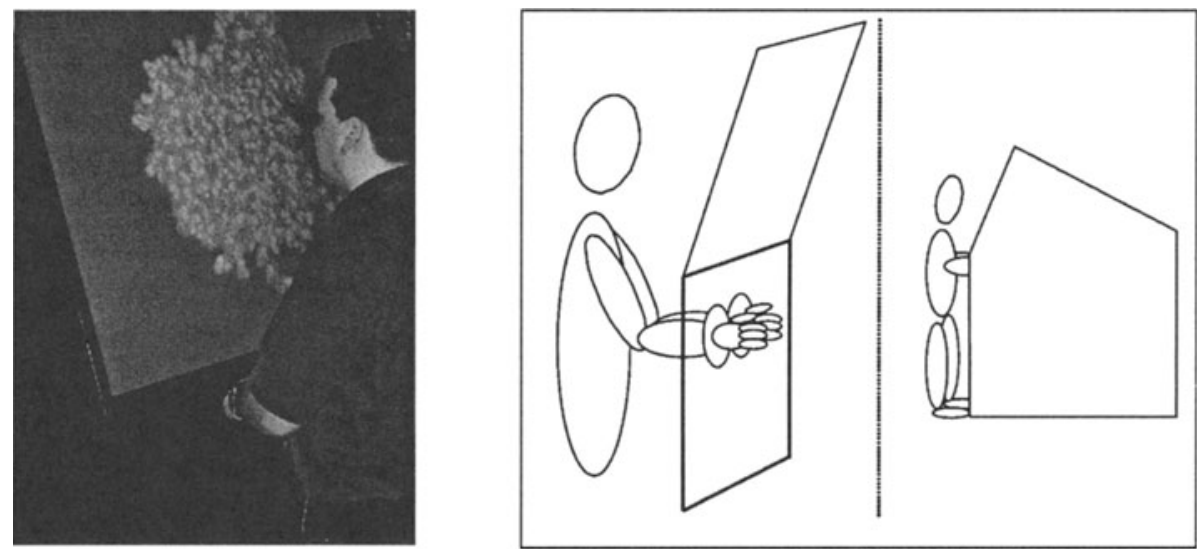

Figure 2: Using the Virtual Glove Box

We used the glove box metaphor to build our Virtual Glove Box. The Virtual Glove Box combines a stereoscopic display with haptic displays. The stereoscopic part consists of a two projector system with polarized lenses which continuously display images for each eye. The user views the scene through matching polarized glasses. Directly below the display frame are two openings through which the user can reach into the box. The user cannot see his real hands but rather a computer generated representation which mirrors the users motions (see Figures 1 and 2).

We achieve haptic feedback by using a Cyber Grasp exoskeleton for each hand [1] (see Figure 3). In this way the user can actively reach into the virtual domain to grab and move the virtual objects there. The whole Virtual Glove Box prototype consists of the glove box mock-up which holds two LCD projectors with polarization filters (one horizontal, the other vertical polarization) and the screen which does not interfere with the polarization of the beams. Inside the box is the emitter of a Polhemus Fastrak magnetical tracker [5] and the two exoskeletons for the hands each equipped with the corresponding receiver for the tracker. In this set-up the position of the hands can be consistently tracked and processed by the application. Both the visual 
and the haptic display is driven by an IBM PC workstation running Microsoft Windows 2000. The computer features an Intense3D Wildcat 4210 graphics card which is able to drive both projectors. One of the technical challenges in this set-up was to avoid interference between the users motions and the optical display.

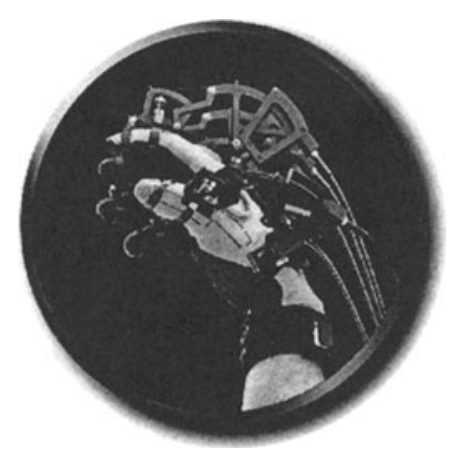

Figure 3: Haptic Feedback Device [1] built in the Glove Box and hidden from the user

\section{SYSTEM INTEGRATION OF THE VIRTUAL GLOVE BOX}

Although the new I/O device offers many benefits, it is unlikely that the Virtual Glove Box supersedes the traditional infrastructure in an enterprise, because of the space requirements of the device and the higher costs compared to conventional hardware. Therefore care must be taken to integrate the new device into existing platforms and structures through dedicated software. It is important that results and models which were built using the Virtual Glove Box are also available to applications which run on traditional and possibly heterogeneous systems.

We address these problems by using an approach derived from methodologies from software engineering: Applications for the Virtual Glove Box are built as frameworks wherein so called components can used to build complex virtual scenes and simulations [2][7]. Frameworks are skeleton applications which provide the adaptor to different hardware configurations or application backgrounds (e.g. Virtual Glove Box or traditional equipment and a development application versus a trade fair presentation). A framework comes to live through components. Components are reusable software modules which encapsulate certain attributes and features of the objects they describe. In the Virtual Glove Box context there 
might be visual and haptic components describing for example pipes for a chemical plant or other non-visual components which encapsulate conventional simulators for example. Since the components are platform and application independent information losses can be minimized.

\section{APPLICATION EXAMPLE}

The Virtual Glove Box enhances the immersion since the haptic exoskeletons which are worn by the user are hidden from view by the apparatus and presents thus a holistic approach for immersive VR devices with haptic feedback.

The Virtual Glove Box offers a multitude of different fields of application in a digital enterprise. The foremost area of application as described above is the planning, modelling and simulation of three dimensional objects from production plants, machinery, pipeline systems etc. to structures on a microscopic level and smaller like molecular structures. The usage is not limited to real objects, abstract systems like work flow diagrams can be experienced likewise.

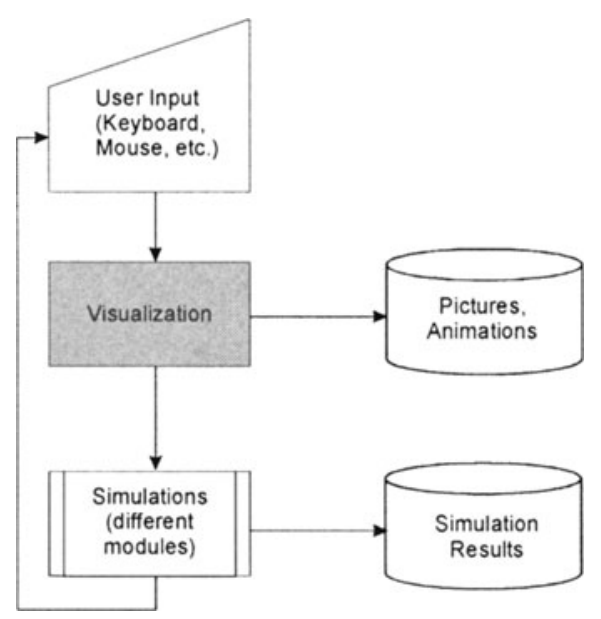

Figure 4: Traditional Approach

A very important aspect of the device is the communication presentation and visualization of new ideas or solutions, which opens the usage of the Virtual Glove Box to a many different sets of users. The expert benefits from the intuitive interaction because he can concentrate on his domain competences (e.g. design or mechanics) rather than learn to interact with complicated authoring systems. The same benefit is shared by users from the 
management, marketing or by customers: things are easier to communicate and to understand if you have a "hands-on "experience.

The usage of a real life interaction metaphor like a glove box drastically increases the user acceptance for such a new device. This is especially true for applications in a digital chemistry or pharmaceutical enterprise, where the glove box metaphor stems from. We demonstrate the usage and the benefits of the Virtual Glove Box device and concept in the field of molecular modelling applications. Molecular modelling describes the technique of designing chemical compounds with the help of the computer. These chemical compound, i.e. molecules etc. are modelled in order to achieve a predefined behaviour. Examples might be the design and research of protein folding structures or the design of specific molecules that interact with such proteins in a desired way (key-lock problem). The traditional notation of chemical structures uses characters and lines to describe a molecule. Such a description is essentially two dimensional. However the molecules and drugs encountered in the biochemistry or pharmaceutical industry cannot be fully described in such a notation since their functions derive from their three dimensional structure. Therefore the primary interest in molecular modelling is the three-dimensional nature of various molecules. In order to visualize such structures for molecular modelling the visualization has to be a $3 \mathrm{D}$ visualization which is expressive for the field of interest.

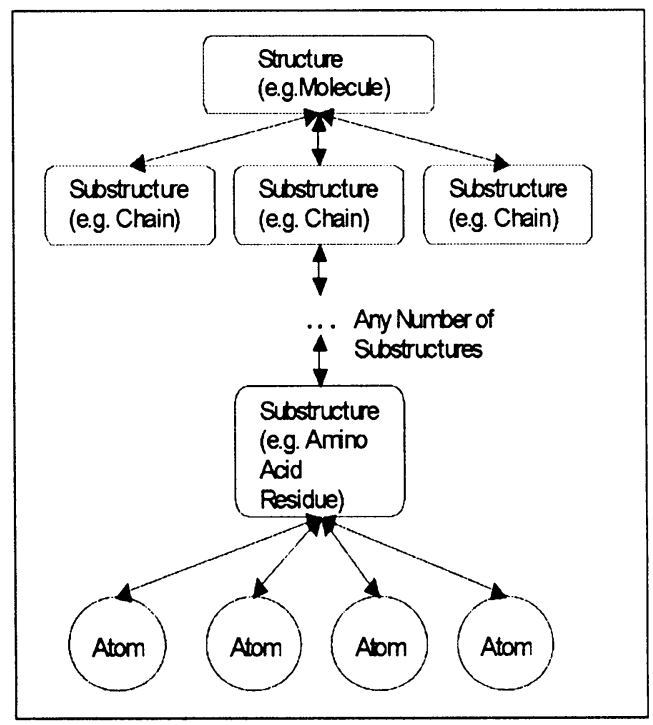

Figure 5: Example Component Hierarchy

In order to improve the process of molecular modelling and to find a solution to stated problems it is necessary to do an analysis of the role of the 
user in a molecular modelling application. The user, mostly a domain expert in the field of chemistry, biology or pharmacy, combines known chemical reagents, parts of molecules or even single atoms to form new structures. For this task exchanged forces or constraints like bond angles etc. have to be taken into account. Hence the composition of the 3D scene is twofold: it is determined partly by physically based behaviour of the entities, partly it depends on the user and his input (see Figure 4). Since the user is working with molecules and atoms as building blocks of the scene, a component based approach is very well suited to the task. In this concept atoms form basic components from which higher level components like molecules can be built (see Figure 5).

The process of molecular modelling is essentially an interactive simulation. The components used in this approach are therefore very similar to those in different simulation centred applications like e.g.. logistic simulations. When the user interacts with the components using the Virtual Glove Box device, he can make use of all aspects of the component, the visual representation, the simulation and the haptic behaviour (see Figure 6). The same components and the results from the work with the Virtual Glove Box can be transferred to another application running on conventional hardware. While the haptic behaviour is still available on the other platform the application framework might not access this feature, because there is no suitable I/O device. In another framework there might be no need for the simulation capabilities but only for the visual representation of the components. Thus we have shown that the Virtual Glove Box component concept adapts very well for different environments and is therefore well suited to be integrated into an established digital enterprise.

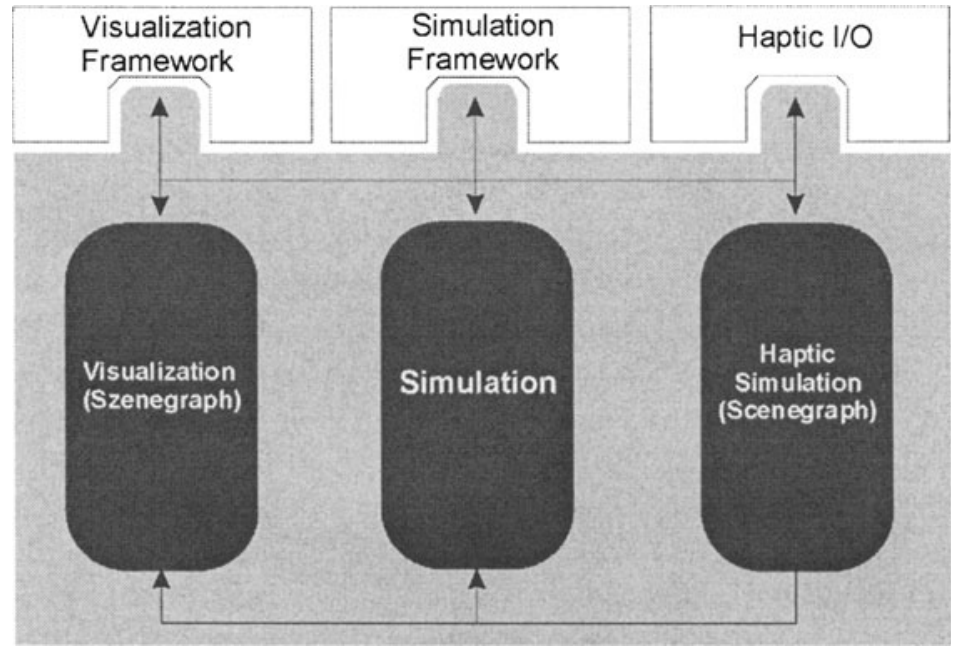

Figure 6: Inner Structure of a Component with Haptic Simulation 


\section{CONCLUSIONS}

In this paper we introduced a new interaction device for manipulating 3D content that responds to the necessities of a digital enterprise. We have shown that it is crucial to design these kind of high-level, holistic devices in order to take a competitive advantage in using $3 \mathrm{D}$ and virtual reality. We identified three main requirements for such an interaction device:

- It has to be designed based on high-level interaction metaphors people are already used to and it has to actively fight acceptance problems

- It has to be holistic and multi-modal

- Not only the hardware-side of the interaction device needs to be taken into account during its design but also the software side (e.g. integration in existing software, application of component and reuse concepts).

If these requirements are met chances are high that productivity can be raised while time can be saved since there is a direct, intuitive interaction and a lower cognitive load associated with the usage of 3D. In addition, a digital enterprise can be achieved as acceptance in dealing with digital data is higher. And the benefits can be used for different groups of users (ranging from technical experts to end customers) and different purposes (ranging from product design to product presentation).

\section{REFERENCES}

[1] CyberGrasp by Virtual Technologies, Inc:

http://www.virtex.com/products/hw_products/cybergrasp.html

[2] R. Doerner, P. Grimm: Three-dimensional Beans - Creating Web content using 3D components in a 3D authoring environment, Proceedings of the Web3D-VRML 2000 fifth symposium on Virtual reality modeling language, Pages $69-74,2000$

[3] R. W. Lindeman, J. L. Sibert and J. K. Hahn: Towards usable VR: an empirical study of user interfaces for immersive virtual environments; Proceeding of the $\mathrm{CHI}$ 99 conference on Human factors in computing systems: the $\mathrm{CHI}$ is the limit, Pages $64-71,1999$, Pittsburgh, PA USA 
[4] V. Luckas, R. Doerner: Experiences from the Future: Using Object-oriented Concepts for 3D Visualization and Validation of Industrial Scenarios, ACM Computing Surveys, Vol. 32, Issue 2, p. 38-44, ACM Press, 2000

[5] Polhemus Fastrak, http://www.polhemus.com/ftrakds.htm

[6] K. Saliburym D. Brook, T. Massie, N. Swarup, C. Zilles: Haptic rendering: programming touch interaction with virtual objects; Proceeding of the 1995 symposium on Interactive 3D graphics, Pages 123-130, 1995, Monterey, CA USA

[7] J. Sametinger: Software Engineering with Reusable Components. Springer Verlag, 1997

[8] M. A. Srinivasan, C. Basdogan: "Haptics in Virtual Environments: Taxonomy, Research Status, and Challenges", Computers and Graphics, Special Issue on Haptic Displays in Virtual Environments, Vol. 21, No. 4, IEEE Press, 1997

[9] Y. Yokokohji, R. Hollis, T. Kanade: Vision-based Visual/Haptic Registratration for WYSIWYF Display, International conference on Intelligent Robots and Systems, IROS '96, Pages 1386-1393, 1996 